\title{
Expression and purification of neurolin immunoglobulin domain 2 from Carrassius auratus (goldfish) in Escherichia coli
}

\author{
Christina Drees $^{\text {a }}$, Claudia A.O. Stürmer ${ }^{\mathrm{b}}$, Heiko M. Möller ${ }^{\mathrm{a}, *}$, Günter Fritz ${ }^{\mathrm{b}, *}$ \\ a Department of Chemistry, University of Konstanz, 78457 Konstanz, Germany \\ ${ }^{\mathrm{b}}$ Department of Biology, University of Konstanz, Universitaetsstrasse 10, Postfach M 665, 78457 Konstanz, Germany
}

\begin{abstract}
The immunoglobulin superfamily protein neurolin plays a central role during differentiation and development of retina ganglion cells in goldfish. As shown in earlier work, blockage of the second immunoglobulin domain (Ig2) of neurolin with domain specific antibodies causes severe pathfinding defects of growing axons in the retina. Thus Ig2 of neurolin was identified as the critical domain for axon guid ance. In the present study we have developed a protocol for expression and purification of neurolin Ig2 suitable for structure analysis, functional studies and ligand identification. Neurolin was expressed in Rosettagami and Origami strains of Escherichia coli which is defi cient in glutathione and thioredoxin reductase facilitating proper formation of the disulfide bond in the cytoplasm. The protein was purified via an $\mathrm{N}$ terminal $\mathrm{His}_{6}$ tag by $\mathrm{Ni}^{2+}$ affinity and size exclusion chromatography. After purification the His 6 tag was cut off with out loss of solubility. Analytical size exclusion chromatography revealed an apparent molecular mass for neurolin Ig2 in agreement with a non covalent homodimer. Analysis of CD and FTIR spectra gave a secondary structure content typical for Ig domains.
\end{abstract}

Keywords: Neurolin; Axonal growth; Axonal regeneration; Immunoglobulin; Neuronal development

Neurolin was identified as a growth-related neuronal cell surface protein in goldfish [1]. It is a $90 \mathrm{kDa}$ glycosylated protein of the immunoglobulin (Ig) superfamily [2,3], consisting of five extracellular Ig domains, a single transmembrane helix, and a highly conserved cytoplasmic domain. Homologs, designated ALCAM, SC1/BEN, DM-GRASP, have been found in human, rodents, and chicken, respectively. Expression of neurolin is upregulated on axons of retinal ganglion cells during growth and axonal regeneration. In the retina neurolin is selectively expressed by the newly differentiated retinal ganglion cells and their growing axons [3]. In an earlier study, neurolin function was probed by in vivo studies with antibodies specifically directed against Ig domains 1, 2, and 3. Blockage of neurolin func-

\footnotetext{
* Corresponding authors. Fax: +49 7531885174 (H.M. Moller), +49 7531882966 (G. Fritz).

E mail addresses: heiko.moeller@uni konstanz.de (H.M. Moller), guenter.fritz@uni konstanz.de (G. Fritz).
}

tion led to severe pathfinding mistakes [4,5]. Instead of growing towards the optic disk, axons left their fascicle of origin and turned around or entirely lost orientation $[4,5]$. It was shown that the second Ig domain of neurolin is preferentially involved in this pathfinding function [4]. Neurolin function is not exclusive for goldfish. In zebrafish, neurolin has a similar function leading to massive defects of neuronal development upon application of neurolin-specific antibodies [6]. Recently, it was reported that a mouse lacking the neurolin-homolog BEN showed retinal pathfinding errors and dysplasia [7]. How neurolin exerts its function in these organisms is not well understood, and several alternative mechanisms are being discussed $[4,5]$. Like its closest homolog ALCAM in mammalia, neurolin might form homo- or heterophilic contacts by cis and/or trans interactions. However, this has not been shown for neurolin so far. The experimental data from in vivo experiments suggest that neurolin-Ig2, which is crucial for pathfinding function, interacts with a soluble or 
membrane-bound guiding component, and a gradient of the guiding molecule might direct axonal growth. Hence, neurolin plays a key role in the development of the visual system and is also involved in regeneration of the fish (goldfish, zebrafish) optic nerve representing a model system for neuronal development and regeneration. Characterizing structure and function of neurolin will improve our understanding of axon guidance and regeneration, particularly since the mammalian central nervous system has lost the capacity of regeneration, e.g. after spinal cord injury or stroke.

Here we report on an efficient protocol for the production of recombinant neurolin-Ig2 in Escherichia coli, which allows to express neurolin-Ig2 for structural characterization by X-ray crystallography and NMR spectroscopy. The expression of neurolin-Ig2 in $E$. coli has the advantage that the protein lacks glycosylation and is amenable for isotope-labeling with ${ }^{15} \mathrm{~N}$ and ${ }^{13} \mathrm{C}$ for multidimensional $\mathrm{NMR}$ experiments or SeMet for phase determination in X-ray crystallography. Furthermore, the recombinant neurolinIg2 preparation is suitable for ligand capture experiments aimed at identifying a soluble guidance compound, and for extensive in vivo experiments which will lead to a better understanding of neurolins function.

\section{Materials and methods}

\section{Bacterial strains}

Escherichia coli JM109 was used for amplification of plasmids and E. coli BL21(DE3) Origami B (Novagen), E. coli Rosettagami BL21(DE3) B, E. coli BL21(DE3) B (Novagen), and E. coli BL21(DE3) Rosetta B (Novagen) were used for recombinant expression of neurolin-Ig2. Competent $E$. coli cells were prepared according to Inou et al. [8].

\section{Cloning}

Cloning of neurolin-Ig2 was performed by Trenzyme (Konstanz, Germany). The cDNA corresponding to the Ig2 domain (amino acid residues 132 228) of goldfish neurolin was amplified by PCR using a plasmid containing the whole cDNA [4]. The primers used for the cloning of neurolin-Ig2 were designed using the nucleotide sequence published in the GenBank database (Accession No. L25056) and contained the two restriction sites NdeI and XhoI. Furthermore, a stop codon was introduced before the XhoI site. The following forward (5'-AAA CAT ATG TCA GCC CCT GTA ATC AAA AAC AAC G-3') and reverse primers (5'-ACT CGA GAT TAC TGG TCG GGG CCC ATC AC-3') were used to amplify neurolin-Ig2 using the following PCR conditions: $2 \mathrm{~min}$ at $94{ }^{\circ} \mathrm{C}$ followed by 30 cycles of $15 \mathrm{~s}$ at $94^{\circ} \mathrm{C}, 30 \mathrm{~s}$ at $60^{\circ} \mathrm{C}$, and $2 \mathrm{~min}$ at $72^{\circ} \mathrm{C}$; the final extension was for $7 \mathrm{~min}$ at $72{ }^{\circ} \mathrm{C}$ using Phusion polymerase (New England Biolabs). The PCR products were analyzed on $1.5 \%$ agarose gel and extracted from the agarose gel using the DNA extraction kit (Quiagen). The DNA was ligated blunt-end into vector pAlli10 (Trenzyme) and the sequence was checked by sequencing using standard M13 forward primer. The resulting pAlli10-neurolin-Ig2 was digested with NdeI and XhoI (New England Biolabs) separated on a 1\% agarose gel and extracted from the agarose gel using QIAquick Gel Extraction Kit (Qiagen). The pET15b vector was digested with the same two enzymes and purified from $1 \%$ agarose gels. The digested insert from pAlli10-neurolin-Ig2 was ligated into the linearized vector $\mathrm{pET} 15 \mathrm{~b}$ at $25^{\circ} \mathrm{C}$ for $5 \mathrm{~min}$ using the Rapid Ligation Kit (Roche Applied Science). Vector pET15b contains the sequence of an N-terminal hexa-histidine tag ( His $_{6}$-tag) to facilitate purification of neurolin-Ig2 and a thrombin-cleavage site for post-translational removal of the $\mathrm{His}_{6}$-tag. The resulting vector pET15b-neurolin-Ig2 was transformed into competent E. coli JM109 cells and amplified.

\section{Expression and purification}

Competent $E$. coli strains were transformed with $5 \mathrm{ng}$ of plasmid DNA. Transformed cells were selected on DYT agar plates containing $100 \mu \mathrm{g} \mathrm{ml}{ }^{1}$ ampicillin (AppliChem), $15 \mu \mathrm{g} \mathrm{ml}^{1}$ kanamycin (AppliChem), for E. coli BL21(DE3) Origami B or additionally $34 \mu \mathrm{g} \mathrm{ml}{ }^{1}$ chloramphenicol (Fluka) for selection of the E. coli Rosettagami BL21(DE3) B strain. A single colony was used to inoculate $25 \mathrm{ml}$ of DYT medium containing $0.2 \%$ glucose and the same amount of antibiotics as listed above. The culture was incubated overnight at $37^{\circ} \mathrm{C}$ and $220 \mathrm{rpm}$. Cells were pelleted by centrifugation at $5000 \mathrm{~g}$ at $4{ }^{\circ} \mathrm{C}$ for $5 \mathrm{~min}$ and the supernatant culture medium was discarded in order to remove extracellular $\beta$-lactamases. The pellet was resuspended in $12 \mathrm{ml}$ fresh DYT medium and $5 \mathrm{ml}$ of this suspension were used to inoculate $500 \mathrm{ml}$ DYT medium. Cells were grown at $37^{\circ} \mathrm{C}$ to an $\mathrm{OD}_{600 \mathrm{~nm}}$ of about 0.6 . Then temperature was decreased to $25^{\circ} \mathrm{C}$ and the culture was grown to an $\mathrm{OD}_{600 \mathrm{~nm}}$ of about 1.2. Expression of neurolin-Ig2 was induced by addition of $0.5 \mathrm{mM} \mathrm{IPTG}^{1}$ (AppliChem) and cells were allowed to grow at $25^{\circ} \mathrm{C}$ for another $1214 \mathrm{~h}$. Cells were harvested by centrifugation at $8000 \mathrm{~g}$ at $4{ }^{\circ} \mathrm{C}$ for $20 \mathrm{~min}$. The cell pellets were used immediately for protein purification or frozen in liquid nitrogen and stored at $-70{ }^{\circ} \mathrm{C}$.

All purification steps were performed at $4{ }^{\circ} \mathrm{C}$. Ten grams of wet weight cells were suspended in ice-cold $40 \mathrm{ml} 50 \mathrm{mM}$ $\mathrm{KH}_{2} \mathrm{PO}_{4}, \mathrm{pH}$ 7.4. Protease inhibitors (Complete, Roche Diagnostics) and $0.4 \mathrm{mM}$ PMSF (AppliChem) were added. In order to remove large DNA fragments released upon cell rupture $5 \mathrm{mM} \mathrm{MgCl}_{2}$ and a spatula tip of DNAse (Roche Diagnostics) were added. Cells were ruptured by three passages through a French pressure cell at

\footnotetext{
${ }^{1}$ Abbreviations used: IPTG, isopropyl $\beta$ D thiogalactopyranoside; FTIR, Fourier transformed infra red.
} 
$138 \mathrm{MPa}$. The lysate was centrifuged for $75 \mathrm{~min}$ at $100,000 \mathrm{~g}$ at $4{ }^{\circ} \mathrm{C}$. The supernatant containing folded neurolin-Ig2 was used for further purification. A Ni-Sepharose Fast Flow column (GE-Healthcare) was equilibrated with 2 column volumes buffer I $\left(50 \mathrm{mM} \mathrm{KH}{ }_{2} \mathrm{PO}_{4}, 300 \mathrm{mM}\right.$ $\mathrm{NaCl}, 10 \mathrm{mM}$ imidazole, $\mathrm{pH}$ 7.4). The supernatant was diluted 25-fold with buffer I and loaded onto the column. After washing with buffer I (3 column volumes), bound protein was eluted in a linear gradient with $50 \mathrm{mM}$ $\mathrm{KH}_{2} \mathrm{PO}_{4}, 300 \mathrm{mM} \mathrm{NaCl}, 0.5 \mathrm{M}$ imidazole, $\mathrm{pH} 7.4$ over 4 column volumes. The eluting fractions were analyzed on Coomassie stained 15\% SDS PAGE [9].

The fractions containing neurolin-Ig2 were combined and concentrated by ultrafiltration and dialyzed against $50 \mathrm{mM}$ Hepes, $2 \mathrm{mM} \mathrm{CaCl}_{2}, 300 \mathrm{mM} \mathrm{NaCl}, \mathrm{pH}$ 7.4. After dialysis the $\mathrm{pH}$ of the protein solution was adjusted to 8.2 by dropwise addition of a $2 \mathrm{M}$ Tris solution. For the proteolytic cleavage of the $\mathrm{His}_{6}$-tag $1.5 \mathrm{U}$ thrombin (GEHealthcare) per $\mathrm{mg}$ neurolin- $\operatorname{Ig} 2$ were added and the solution was incubated at $30{ }^{\circ} \mathrm{C}$ for $8 \mathrm{~h}$. The cleaved $\mathrm{His}_{6}$-tag and uncut protein was removed by passage over His-Trap HP column ( $5 \mathrm{ml}$, GE-Healthcare) equilibrated in $50 \mathrm{mM}$ $\mathrm{KH}_{2} \mathrm{PO}_{4}, 300 \mathrm{mM} \mathrm{NaCl}, \mathrm{pH}$ 7.4. The protein was concentrated and loaded onto a Superdex $75(1.6 \mathrm{~cm} \times 60 \mathrm{~cm}$, GE-Healthcare) column equilibrated in the same buffer.

\section{Analytical methods}

Analytical size exclusion chromatography

Analytical size exclusion chromatography was performed on a Superdex $75(1.0 \mathrm{~cm} \times 30 \mathrm{~cm}$, GE-Healthcare $)$ equilibrated in $50 \mathrm{mM} \mathrm{KH} \mathrm{PO}_{4}, 300 \mathrm{mM} \mathrm{NaCl}$, pH 7.4. The column was calibrated with BSA $(66 \mathrm{kDa})$, carbonic anhydrase $(29 \mathrm{kDa})$, cytochrome $c(12.4 \mathrm{kDa})$, and aproti$\operatorname{nin}(6.5 \mathrm{kDa})$ (Sigma) in the same buffer.

\section{Protein determination}

The concentration of pure $\mathrm{His}_{6}$-neurolin-Ig2 or neurolin-Ig2 was determined photometrically using an extinction coefficient $\varepsilon_{280 \mathrm{~nm}} \quad 7115 \mathrm{M}^{1} \mathrm{~cm}^{1}$ as calculated from the content of aromatic residues and the disulfide bond (http://www.expasy.ch/tools/protparam.html). Optical spectra were recorded on a Cary 50 spectrophotometer (Varian) and corrected by subtraction of a blank spectrum obtained with pure buffer.

\section{Thiols}

Analysis of free thiols was performed according to [10].

\section{Mass determination}

The mass of $\mathrm{His}_{6}$-neurolin-Ig2 and neurolin-Ig2 protein was determined by ESI mass spectrometry on a Q-TOF Ultima API (Waters). For reduction of the disulfide bond the protein was incubated in $50 \mathrm{mM}$ Tris $\mathrm{Cl}, \mathrm{pH} 8.0$, $4 \mathrm{M}$ Guanidinium-hydrochloride and $100 \mathrm{mM}$ DTT for 30 min at $35^{\circ} \mathrm{C}$. The samples were purified on a ZipTip and measured in $50 \%$ acetonitrile $/ 0.2 \%$ formic acid $(\mathrm{pH}$
2). Mass spectra (neutral masses) were deconvoluted using the MaxEnt1 software. The theoretical mass was calculated from the primary sequence of the protein encoded on the plasmid (http://www.expasy.ch/tools/protparam.html).

\section{FTIR spectroscopy}

The concentration of neurolin-Ig2 was $50 \mu \mathrm{M}$. FTIR spectra were recorded at $20^{\circ} \mathrm{C}$ on a Bruker Tensor 27 instrument accumulating 1024 scans at a resolution of $2 \mathrm{~cm}^{1}$; spectra of buffer were recorded under the same conditions and subtracted from the sample spectra. The position of individual IR transitions in the amide I region between 1700 and $1600 \mathrm{~cm}^{1}$ were resolved by second order derivative spectra. For protein secondary structure analysis the spectra were fitted with a set of 12 Gaussian line shaped transitions.

\section{Circular dichroism}

Far UV circular dichroism spectra of neurolin-Ig2 (180 $260 \mathrm{~nm}$ ) were measured on a JASCO J-715 instrument using quartz cells with 1 and $0.1 \mathrm{~mm}$ path length. The main compartment of the instrument was flushed with dry nitrogen gas during the measurement. Neurolin-Ig2 was $0.8 \mathrm{mg} /$ $\mathrm{ml}$ in $50 \mathrm{mM}$ acetate, $\mathrm{pH} 4.5$ or $50 \mathrm{mM} \mathrm{KH}_{2} \mathrm{PO}_{4}, 300 \mathrm{mM}$ $\mathrm{NaCl}, \mathrm{pH}$ 7.4. Six spectra were averaged and corrected by subtraction of a blank spectrum obtained with pure puffer. The secondary structure content from the CD spectrum was calculated using the program CDNN $[11,12]$.

\section{Dot blot analysis}

Dots of $2 \mu 1$ of protein solution were placed on a cellulose nitrate membrane (Schleicher \& Schuell). The following solutions were used: neurolin-Ig2 $(0.26 \mathrm{mg} / \mathrm{ml})$, retina lysate ( 1 retina of goldfish lysed in $20 \mathrm{mM}$ Tris, $150 \mathrm{mM}$ $\mathrm{NaCl}, 1 \%$ Triton X-100, 0.1\% SDS). Every solution was applied pure and diluted 1:10 and 1:100 in buffer. The membrane was blocked with Roti-Block (Roth) for $30 \mathrm{~min}$ at room temperature. The membrane was washed twice and incubated overnight at $4{ }^{\circ} \mathrm{C}$ with primary antibody (mouse anti-neurolin-Ig2 monoclonal antibody, N518) [4] diluted 1:2000 in PBS containing Roti-Block. Afterwards the membrane was washed 3 times with PBS containing $0.05 \%$ Tween at room temperature and incubated with secondary antibody (anti-mouse IgG peroxidase conjugate, Sigma), diluted 1:10,000 in $5 \mathrm{ml}$ PBS containing Roti-Block. The membrane was washed with $4 \mathrm{ml}$ PBS containing $0.05 \%$ Tween and the dot blot was stained with SIGMAFAST DAB (Sigma).

\section{Homology modelling}

A 3D model of neurolin-Ig2 was built using MODELLER [13,14] and PHYRE [15,16] web service (http:// www.sbg.bio.ic.ac.uk/phyre/). Template structures for molecular modeling were selected by BLAST $[17,18]$ searches using BLOSUM 62 matrix. Template structures were selected using a cut-off in BLAST hits with an $E$ - 
value $\leq 0.001$ and a sequence identity of $\sim 20 \%$. The highest homology was observed for an Ig domain of the intercellular adhesion molecule-2 (ICAM-2) [19] and a model of neurolin-Ig2 was built using the X-ray structure of ICAM2 at $2.2 \AA$ resolution (pdb code 1zxq) as template. The model was evaluated by visual inspection using the programs SYBYL7.2 (Tripos Inc., St. Louis, MO, USA) and MolMol [20]. The glycosylated homology model was generated within SYBYL7.2 by attaching an N-linked complex-type nona-saccharide to the side chain of Asn171. The coordinates and orientation of the glycan were taken from the $3 \mathrm{D}$ structure of human chorionic gonadotropin (pdb code 1hd4) [21].

\section{Results and discussion}

\section{Expression of recombinant $\mathrm{His}_{6}$-neurolin-Ig2 in E. coli Origami strain}

Full length neurolin is a transmembrane protein with five extracellular Ig domains. Cell surface receptors of the Ig superfamily are very often challenging with regard to heterologous expression of the entire proteins or fragments thereof. Major reasons are the introduction of disulfide bonds which are required for folding and stability of $\mathrm{Ig}$ domains and glycosylation sometimes important for the solubility of the protein. The disulfide bond formation and glycoslyation take place in the endoplasmatic reticulum of eukaryotes. Prokaryotes such as E. coli lack such a complex compartment and utilize other mechanisms for disulfide bond formation in secreted proteins. A further point is applicable to extracellular domains of membrane proteins in general. In their natural environment, i.e. when anchored to the membrane, the extracellular portions of membrane proteins are very limited in terms of diffusion and possibility to form intermolecular contacts compared to an isolated domain in solution. This might be the reason for the frequent observation that the isolated extracellular portions of membrane receptors aggregate and are insoluble even at low concentrations. Nevertheless, due to potentially high yields at low cost, bacterial expression is still the first choice when domains of membrane proteins are produced for structural studies. In the case of neurolin, proper formation of a disulfide bond between Cys154 and Cys 217 is of major concern.

Proteins containing disulfide bridges often turn out to be a challenge concerning expression in E. coli. Disulfides are introduced if the protein is secreted to the periplasm [22], however very often the yield is lower compared to expression in the cytoplasm. On the other hand the low intracellular redox potential disfavors the oxidation of thiols to disulfides. Very often, proteins that require disulfide bridges for structural integrity are thus found misfolded and aggregated in inclusion bodies. E. coli Origami strains are specifically designed to allow intracellular disulfide formation [23]. By knocking-out the genes for thioredoxin reductase and glutathione oxidoreductase the intracellular redox potential is increased such that formation of disulfide bridges can take place. Expression of proteins containing disulfide bonds in these mutant strains results generally in higher yields of active protein compared to the secretion of expressed protein into the periplasm [23]. We expressed neurolin-Ig2 in E. coli Origami and Rosettagami strains that carries an additional plasmid coding for tRNA for seven rare codons in E. coli. Expression at $37^{\circ} \mathrm{C}$ gave rather low yields of soluble protein and most neurolinIg2 was present in inclusion bodies. Expression yields were increased using DYT supplemented with sodium phosphate as a buffer and additional phosphate source, decrease of the expression temperature to $25^{\circ} \mathrm{C}$. Furthermore, addition of $0.21 \%$ glucose to the medium suppressed leaky induction of the T7 polymerase and increase of induction time to $1214 \mathrm{~h}$ improved expression significantly. One liter of culture yielded $914 \mathrm{~g}$ wet weight cells for both the Origami and the Rosettagami strain. Both strains showed high expression of neurolin-Ig2. Approximately $50 \%$ of the protein was present as insoluble inclusion bodies (Fig. 1A). Nevertheless, the yield of soluble His $_{6}$-neurolin-Ig2 and tag-less neurolin-Ig2 was typically 21 and $16 \mathrm{mg}$ soluble protein per liter culture, respectively (Table 1). Expression in the corresponding isogenic strains without altered redox environment at $37^{\circ} \mathrm{C}$ or $25^{\circ} \mathrm{C}$ gave no detectable soluble protein (Fig. 1B).

\section{Neurolin-Ig2 forms a non-covalent homodimer}

Analytical size exclusion chromatography of $\mathrm{His}_{6}$-neurolin-Ig2 and tag-less neurolin-Ig2 showed that both proteins eluted clearly at higher apparent molecular mass than the marker protein cytochrome $c(12.4 \mathrm{kDa})$ (Fig. 2A). Calibration of the column revealed molecular masses of $25 \pm 2 \mathrm{kDa}$ and $23 \pm 2 \mathrm{kDa}$ for $\mathrm{His}_{6}$-neurolin-

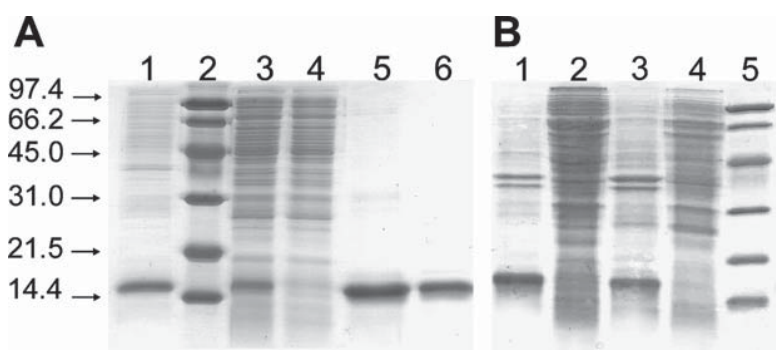

Fig. 1. (A) Purification of $\mathrm{His}_{6}$ neurolin Ig2 from E. coli BL21(DE3) Origami B. The following samples were loaded onto SDS PAGE: lane 1, the pellet after ultracentrifugation contained large amounts of $\mathrm{His}_{6}$ neurolin Ig2 as inclusion bodies; lane 2 molecular weight marker; lane 3, supernatant after ultracentrifugation; lane 4 , protein not bound to $\mathrm{Ni}^{2+}$ Sepharose column; lane 5, eluate of $\mathrm{Ni}^{2+}$ Sepharose column containing soluble $\mathrm{His}_{6}$ neurolin Ig2; lane 6, pure His ${ }_{6}$ neurolin Ig2 after size exclusion chromatography. (B) Expression of $\mathrm{His}_{6}$ neurolin $\mathrm{Ig} 2$ in E. coli BL21(DE3) B or E. coli BL21(DE3) Rosetta B gave only insoluble protein. Lane 1, pellet of E. coli BL21(DE3) Rosetta B: lane 2, soluble fraction $E$. coli BL21(DE3) Rosetta B; lane 3, pellet of $E$. coli BL21(DE3) $\mathrm{B}$; lane 4, soluble fraction of $E$. coli BL21(DE3) B; lane 5, molecular weight marker. 
Table 1

Purification of recombinant neurolin $\mathrm{Ig} 2$ from $1 \mathrm{~L}$ culture of $E$. coli BL21(DE3) Origami B

\begin{tabular}{lcl}
\hline Purification step & Total protein $(\mathrm{mg})$ & Neurolin $(\mathrm{mg})$ \\
\hline Crude extract & 1600 & n.d. \\
Ultracentrifugation, & 700 & $\sim 70$ \\
$\quad$ soluble fraction & & \\
$\mathrm{Ni}^{2+}$ Sepharose & 40 & 36 \\
Superdex 75 & 21 & 21 (with $\mathrm{His}_{6}$ tag)
\end{tabular}

For production of tag less neurolin Ig2 the His6 tag is removed after $\mathrm{Ni}^{2+}$ Sepharose step

Thrombin digest

His Trap HP

Superdex 75

$\begin{array}{ll}35 & 32 \\ 24 & 24 \\ 16 & 16\end{array}$

n.d., not determined.

Ig2 and tag-less neurolin-Ig2, respectively. These masses are in good agreement with the formation of a neurolinIg2 dimer. The dimer was rather stable and did not dissociate even at very low protein concentrations $(\leqslant 5 \mu \mathrm{M})$ in size exclusion chromatography. The mass spectrometric analysis had already shown that the protein does not form a covalent dimer connected by a disulfide. In order to corroborate these results we checked the prepared neurolinIg2 for free cysteine thiols which might be present if a dimeric protein connected by one disulfide bridge has formed. However, no thiols were detected. Furthermore, the oxidation state of the cysteines and the presence of putative covalently linked dimers of neurolin-Ig2 were checked by non-reducing SDS PAGE. His ${ }_{6}$-neurolin-Ig2 reduced by DTT showed one band running slightly above the $14.4 \mathrm{kDa}$ marker (Fig. 2B). In non-reducing SDS PAGE again only one band was detected showing that no disulfide-bridged dimers occur. This band was running at an apparent smaller molecular mass slightly below the $14.4 \mathrm{kDa}$ marker (Fig. 2B), indicating a more compact shape of the protein, which is typical for disulfide containing proteins.

\section{Biochemical and biophysical characterization}

The mass of His $_{6}$-neurolin- $\mathrm{Ig} 2$ was calculated to 12815.4 Da. Mass spectrometric analysis revealed a major peak with a mass of $12684.0 \mathrm{Da}$ (Fig. 2C) showing that the N-terminal methionine was cleaved off (theoretical mass 12686.2 Da). The observed mass difference of $2 \mathrm{Da}$ compared to the mass calculated from the sequence without $\mathrm{N}$-terminal methionine is evidence for the formation of a disulfide bond. Cleavage of the $\mathrm{His}_{6}$-tag gave a protein with a mass of 10933.3 Da (theoretical mass 10935.3 Da). Reduction of neurolin-Ig2 resulted in a mass of 10935.1 Da, as expected for the reduced protein (Fig. 2D).
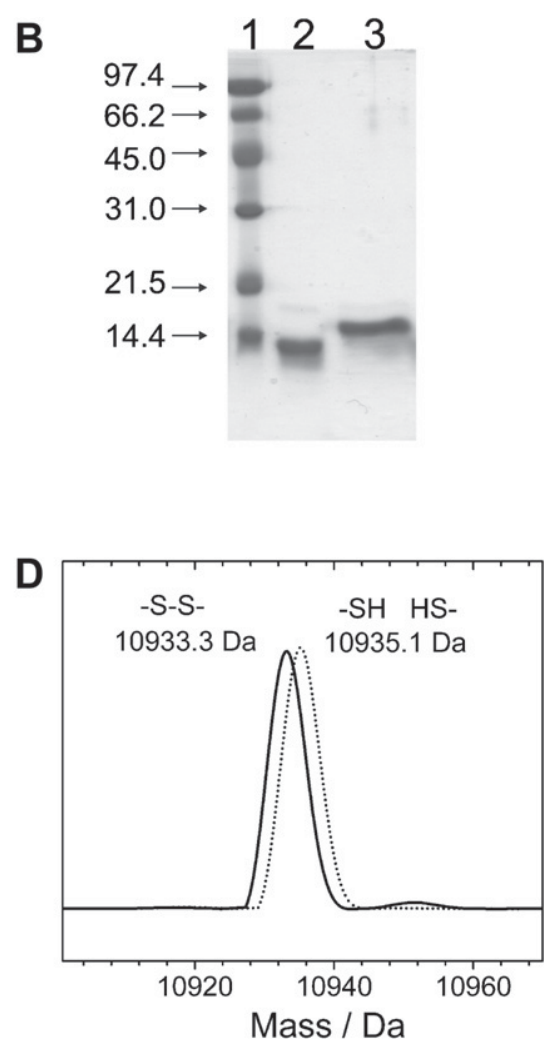

Fig. 2. (A) Analytical size exclusion chromatography of neurolin Ig2. Neurolin Ig2 eluted as a single peak. The elution volumes of marker proteins with known molecular mass are indicated by arrows. (B) SDS PAGE of His ${ }_{6}$ neurolin Ig2: lane 1, marker proteins; lane 2, His 6 neurolin Ig2 not reduced containing a disulfide bond; lane 3, His 6 neurolin Ig2 reduced by DTT in sample buffer. (C) ESI mass spectrometric analysis of His 6 neurolin Ig2. (D) ESI mass spectrometric analysis of neurolin $\operatorname{Ig} 2$ as isolated and neurolin $\operatorname{Ig} 2$ after reduction with DTT. 


\section{Secondary structure content}

The secondary structure content of neurolin-Ig2 was analyzed by CD and FTIR spectroscopy (Fig. 3). Accumulation of a large number of spectra improved the signalto-noise ratio sufficiently so that the positions of single transitions could be resolved from the spectral envelope. A total of 12 Gaussian line-shaped transitions gave an excellent fit of the spectrum between 1700 and $1600 \mathrm{~cm}^{1}$.

Eight out of 12 transitions could be assigned to contributions of $\alpha$-helix $\left(1653 \mathrm{~cm}^{1}\right), \quad \beta$-sheet $\left(1626 \mathrm{~cm}^{1}\right.$, $\left.1637 \mathrm{~cm}^{1}\right)$, $\beta$-turn $\left(1616 \mathrm{~cm}^{1}, 1683 \mathrm{~cm}^{1}\right)$, turns/loops $\left(1659 \mathrm{~cm}^{1}, 1670 \mathrm{~cm}^{1}\right)$, and random coil $\left(1647 \mathrm{~cm}^{1}\right)$ (Table 2). Four bands at 1600, 1610, 1696, and $1706 \mathrm{~cm}^{1}$ were assigned to contributions of carboxylate sidechains with partial protonation [24,25]. Integration of the eight bands containing information about the secondary structure revealed 3\% $\alpha$-helix, $37 \% \beta$-sheet, $17 \% \beta$-turns, and $39 \%$ random coil (Table 3 ).

The CD spectrum of neurolin-Ig2 was typical for proteins with high $\beta$-sheet content (Fig. 3B). Analysis of the CD spectrum by CDNN $[11,12]$ gave a similar secondary structure content as FTIR: 10\% $\alpha$-helix, 33\% $\beta$-sheet, $22 \% \beta$-turn, and $36 \%$ random coil (Table 3 ). The estimates of the secondary structure content by both spectroscopic techniques, FTIR and CD, are in good agreement. There are some small deviations especially in the estimate of the $\alpha$-helical content by both methods. Whereas FTIR points towards the presence of just a helical turn, the analysis of the CD spectrum supports the presence a small helix. However, in the analysis of $\beta$-sheet rich proteins FTIR is superior to $\mathrm{CD}$ and gives better estimates of the secondary structure. In summary, our data show that neurolin-Ig2 adopts a fold rich in $\beta$-sheet typical for an Ig domain protein.

\section{Recognition by a neurolin-Ig2-specific monoclonal antibody}

The monoclonal antibody N518 specifically recognizes Ig domain 2 of neurolin in vivo and causes severe path-find-
Table 2

Component analysis of the FTIR spectrum of neurolin Ig2 and assign ment of secondary structure

\begin{tabular}{ll}
\hline Amide I bands $\left(\mathrm{cm}^{1}\right)$ & Assigned secondary structure \\
\hline 1600 & \\
1610 & \\
1616 & $\beta$ Turn \\
1626 & $\beta$ Sheet \\
1637 & $\beta$ Sheet \\
1647 & Random coil \\
1653 & $\alpha$ Helix \\
1659 & Turn/loop \\
1670 & Turn/loop \\
1683 & $\beta$ Turn \\
1696 & \\
1706 & \\
\hline
\end{tabular}

Table 3

Secondary structure content estimates of neurolin Ig2 from FTIR and CD spectroscopic analysis

\begin{tabular}{lll}
\hline $\begin{array}{l}\text { Secondary structure } \\
\text { type }\end{array}$ & $\begin{array}{l}\text { FTIR } \\
\text { Component } \\
\text { analysis }\end{array}$ & $\begin{array}{l}\text { CD } \\
\text { Deconvolution with } \\
\text { CDNN }\end{array}$ \\
\hline$\alpha$ Helix (\%) & 3.4 & 9.8 \\
$\beta$ Sheet (\%) & 37.4 & 33.3 \\
$\beta$ Turn $(\%)$ & 17.3 & 22.5 \\
Random coil/turns $(\%)$ & 39.2 & 35.8 \\
\hline
\end{tabular}

ing errors of the growing axon. It was concluded that it blocks directly the interaction of neurolin with its binding partner and that the epitope might represent a part of a protein interaction site [4]. Therefore the antibody represents an important tool to test whether recombinant neurolin-Ig2 displays properties which might be required for recognition of its putative binding partner. We could show that the antibody recognizes recombinant $\mathrm{His}_{6}$-neurolinIg2 (Fig. 4). This result implies that antibody N518 does not recognize the glycosylation in native neurolin-Ig2. Furthermore, the epitope is not disturbed by the engineered His $_{6}$-tag. This might enable us to use immobilized His $_{6}{ }^{-}$
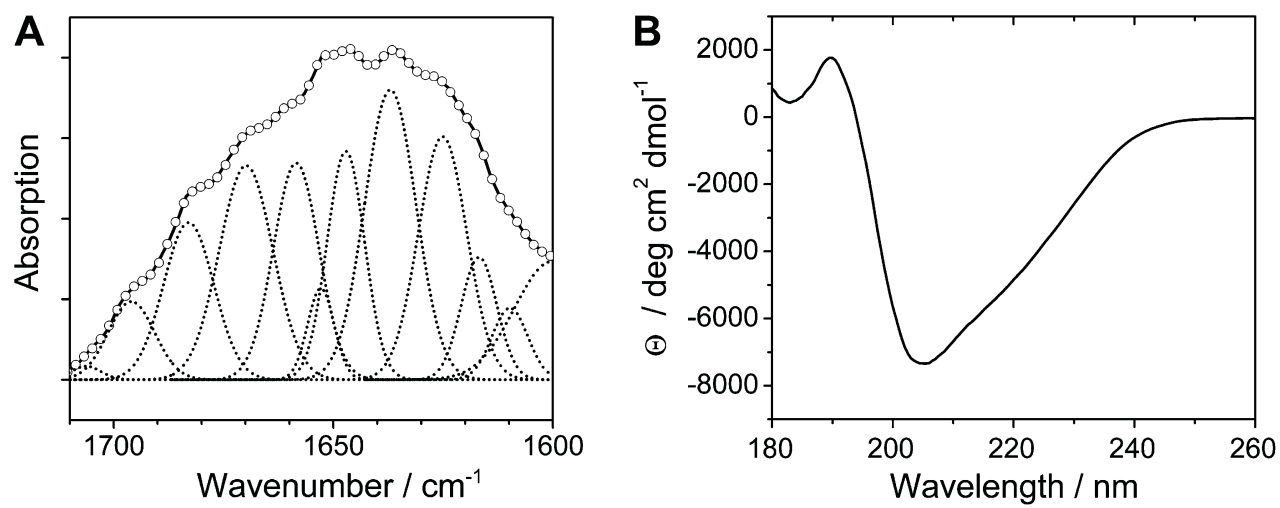

Fig. 3. Secondary structure analysis of $\mathrm{His}_{6}$ neurolin Ig2 by FTIR and CD spectroscopy. (A) FTIR spectrum of the amide I band of neurolin Ig2. The experimental spectrum is shown as solid line, overlaid by the sum (white circles) of 12 Gaussian line shaped transitions shown underneath (dotted lines) The eight central transitions were assigned to contributions from secondary structure elements (Table 2). (B) CD spectrum of neurolin Ig2. The shape of the $\mathrm{CD}$ spectrum reveals high content of $\beta$ sheet in the secondary structure (see Table 3 ). 


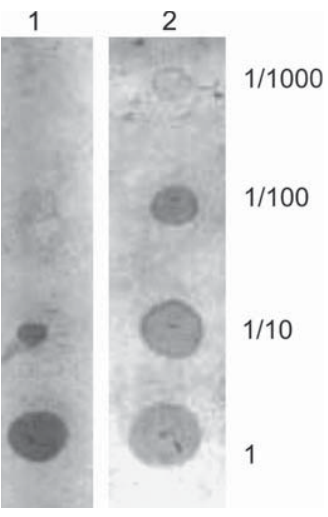

Fig. 4. Recognition of $\mathrm{His}_{6}$ neurolin Ig2 by an antibody that binds to native Ig2 in entire neurolin in vivo. Dot blot of recombinant $\mathrm{His}_{6}$ neurolin Ig2 (1) and retina lysates (2) stained with the anti neurolin Ig2 antibody N518. From bottom to top protein at different dilutions was applied.

neurolin-Ig2 for affinity purification of the putative binding partner.

\section{Modeling}

In order to identify a potential dimerization site of neurolin-Ig2 and check the influence of glycosylation at Asn171 a molecular model was built (Fig. 5). The low sequence identity of neurolin-Ig2 to most Ig domains of known structure made the selection of a template structure difficult. Therefore a series of models of neurolin-Ig2 were built by the PHYRE fold recognition server. Highest homology was observed to ICAM-2 [19] with an $E$-value of

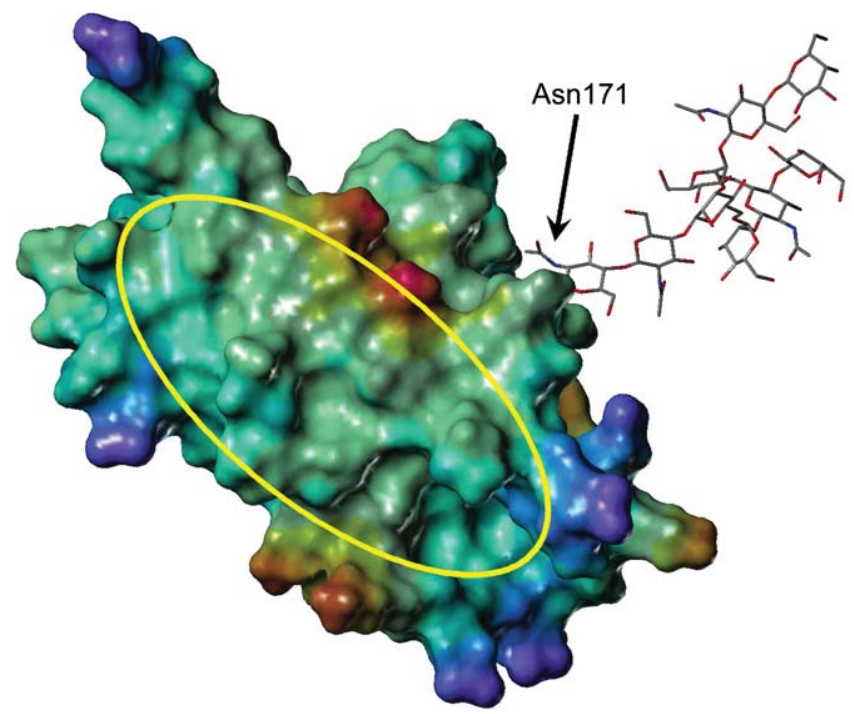

Fig. 5. Homology model of neurolin Ig2. A homology model of neurolin Ig2 comprising residues 134226 was built using the structure of ICAM 2 as template ( $\mathrm{pdb}$ code: 1zxq). The molecular surface of neurolin Ig2 is shown colored according to the electrostatic potential. Positively charged patches are depicted in red, negatively charged patches in blue and neutral areas in green. The glycosylation at Asn171 is shown in stick type. The yellow oval indicates a potential dimerization plane.
$2.4 \times 10^{4}$. The model, derived from the alignment with high estimated precision (95\%), was selected for further analysis. The proper stereochemistry of the model was verified using PROCHECK [26]. Interestingly, the glycosylation site at Asn171 is conserved in ICAM-2 supporting that both proteins adopt a similar structure.

According to the characterization of neurolin glycosylation [27] we attached a complex-type nona-saccharide to Asn171 in order to model glycosylated neurolin. The conformation of the $\mathrm{N}$-glycan was taken from the NMR stucture of human hCG [21]. The glycosylation site in the model of neurolin-Ig2 was well accessible for this glycosylation and no sterical hindrance of the sugar moieties was observed.

Frequently glycosylation of extracellular proteins covers hydrophobic areas thereby increasing the solubility of the glycosylated protein. Analysis of the surface charge around the glycosylation site in our neurolin-Ig2 model revealed predominantly charged areas. Thus, the missing glycosylation of recombinant neurolin- $\operatorname{Ig} 2$ expressed in $E$. coli should not affect solubility. However, a large hydrophobic plane, formed by side chains of Pro2, Val3, Ile4, Val24, Ala27, and the backbone of residues Glu82 to Va190, was observed (Fig. 5). This area is distant from the site of glycosylation and might serve as dimerisation plane of the neurolin-Ig2 dimer as revealed by size exclusion chromatography (Fig. 2A).

\section{Acknowledgments}

We thank Serge Chesnov at the Functional Genomics Center Zurich for the mass spectrometric analysis of His $_{6}$-neurolin-Ig2. We thank Christina Munderloh for providing retina lysate, which was used for the dot blot experiment.

\section{References}

[1] K.A. Paschke, F. Lottspeich, C.A. Stuermer, Neurolin, a cell surface glycoprotein on growing retinal axons in the goldfish visual system, is reexpressed during retinal axonal regeneration, J. Cell Biol. 117 (1992) 863875 .

[2] T. Brummendorf, F.G. Rathjen, Cell adhesion molecules 1: immu noglobulin superfamily, Protein Profile 2 (1995) 9631108.

[3] C.A. Stuermer, M. Bastmeyer, The retinal axon's pathfinding to the optic disk, Prog. Neurobiol. 62 (2000) 197214.

[4] C.A. Leppert, H. Diekmann, C. Paul, U. Laessing, M. Marx, M. Bastmeyer, C.A. Stuermer, Neurolin Ig domain 2 participates in retinal axon guidance and $\mathrm{Ig}$ domains 1 and 3 in fasciculation, J. Cell Biol. 144 (1999) 339349.

[5] H. Ott, M. Bastmeyer, C.A. Stuermer, Neurolin, the goldfish homolog of DM GRASP, is involved in retinal axon pathfinding to the optic disk, J. Neurosci. 18 (1998) 33633372.

[6] H. Ott, H. Diekmann, C.A. Stuermer, M. Bastmeyer, Function of Neurolin (DM GRASP/SC 1) in guidance of motor axons during zebrafish development, Dev. Biol. 235 (2001) 8697.

[7] J.A. Weiner, S.J. Koo, S. Nicolas, S. Fraboulet, S.L. Pfaff, O. Pourquie, J.R. Sanes, Axon fasciculation defects and retinal dysplasias in mice lacking the immunoglobulin superfamily adhesion molecule BEN/ALCAM/SC1, Mol. Cell. Neurosci. 27 (2004) 5969. 
[8] H. Inoue, H. Nojima, H. Okayama, High efficiency transformation of Escherichia coli with plasmids, Gene 96 (1990) 2328.

[9] U.K. Laemmli, Cleavage of structural proteins during the assembly of the head of bacteriophage T4, Nature 227 (1970) 680685

[10] G. Fritz, C.W. Heizmann, P.M. Kroneck, Probing the structure of the human $\mathrm{Ca}^{2+}$ and $\mathrm{Zn}^{2+}$ binding protein S100A3: spectroscopic investigations of its transition metal ion complexes, and three dimensional structural model, Biochim. Biophys. Acta 1448 (1998) 264276.

[11] G. Bohm, CDNN 2.1 CD Spectra Deconvolution, Halle, 1997.

[12] G. Bohm, R. Muhr, R. Jaenicke, Quantitative analysis of protein far UV circular dichroism spectra by neural networks, Protein Eng. 5 (1992) 191195

[13] A. Fiser, A. Sali, ModLoop: automated modeling of loops in protein structures, Bioinformatics 19 (2003) 25002501.

[14] A. Sali, L. Potterton, F. Yuan, H. van Vlijmen, M. Karplus, Evaluation of comparative protein modeling by MODELLER, Proteins 23 (1995) 318326

[15] P.A. Bates, L.A. Kelley, R.M. MacCallum, M.J. Sternberg, Enhance ment of protein modeling by human intervention in applying the automatic programs 3D JIGSAW and 3D PSSM, Proteins (Suppl. 5) (2001) 3946.

[16] L.A. Kelley, R.M. MacCallum, M.J. Sternberg, Enhanced genome annotation using structural profiles in the program 3D PSSM, J. Mol. Biol. 299 (2000) 499520.

[17] S.F. Altschul, J.C. Wootton, E.M. Gertz, R. Agarwala, A. Morgulis, A.A. Schaffer, Y.K. Yu, Protein database searches using composi tionally adjusted substitution matrices, FEBS J. 272 (2005) 5101 5109.

[18] Y.K. Yu, E.M. Gertz, R. Agarwala, A.A. Schaffer, S.F. Altschul, Retrieval accuracy, statistical significance and compositional similar ity in protein sequence database searches, Nucleic Acids. Res. 34 (2006) 59665973.
[19] J.M. Casasnovas, T.A. Springer, J. liu, S.C. Harrison, J. Wang, Crystal structure of ICAM 2 reveals a distinctive integrin recognition surface, Nature 387 (1997) 312315.

[20] R. Koradi, M. Billeter, K. Wuthrich, MOLMOL: a program for display and analysis of macromolecular structures, J. Mol. Graph. 14 (1996) 5155,2932

[21] P.J. Erbel, Y. Karimi Nejad, J.A. van Kuik, R. Boelens, J.P. Kamerling, J.F. Vliegenthart, Effects of the $\mathrm{N}$ linked glycans on the $3 \mathrm{D}$ structure of the free alpha subunit of human chorionic gonado tropin, Biochemistry 39 (2000) 60126021.

[22] H.V. Le, P.P. Trotta, Purification of secreted recombinant proteins from Escherichia coli, Bioprocess Technol. 12 (1991) 163181.

[23] P.H. Bessette, F. Aslund, J. Beckwith, G. Georgiou, Efficient folding of proteins with multiple disulfide bonds in the Escherichia coli cytoplasm, Proc. Natl. Acad. Sci. USA 96 (1999) 13703 13708.

[24] K. Fahmy, F. Jager, M. Beck, T.A. Zvyaga, T.P. Sakmar, F. Siebert, Protonation states of membrane embedded carboxylic acid groups in rhodopsin and metarhodopsin II: a Fourier transform infrared spectroscopy study of site directed mutants, Proc. Natl. Acad. Sci. USA 90 (1993) 1020610210.

[25] F. Jager, K. Fahmy, T.P. Sakmar, F. Siebert, Identification of glutamic acid 113 as the Schiff base proton acceptor in the metarhodopsin II photointermediate of rhodopsin, Biochemistry 33 (1994) 1087810882.

[26] R.A. Laskowski, M.W. MacArthur, D.S. Moss, J.M. Thornton, PROCHECK: a program to check the stereochemical quality of protein structures, J. Appl. Crystallogr. 26 (1993) 283291.

[27] T. Denzinger, H. Diekmann, K. Bruns, U. Laessing, C.A. Stuermer, M. Przybylski, Isolation, primary structure characterization and identification of the glycosylation pattern of recombinant goldfish neurolin, a neuronal cell adhesion protein, J. Mass Spectrom. 34 (1999) 435446. 